\title{
Clinical outcome and predictive factors for docetaxel and epirubicin neoadjuvant chemotherapy of locally advanced breast cancer
}

\author{
Hye Sung Won 1,*, Yong Seok Kim², \\ and Dong Soo Lee ${ }^{6}$
}

\begin{abstract}
Departments of ${ }^{1}$ Internal Medicine, ${ }^{2}$ Surgery, ${ }^{3}$ Hospital Pathology, ${ }^{4}$ Nuclear Medicine, ${ }^{5}$ Radiology, and ${ }^{6}$ Radiation Oncology, Uijeongbu St. Mary's Hospital, College of Medicine, The Catholic University of Korea, Uijeongbu, Korea
\end{abstract}

Received: January 22, 2019

Revised : July 3, 2019

Accepted: July 3, 2019

\section{Correspondence to}

Jeong Soo Kim M.D.

Department of Surgery,

Uijeongbu St. Mary's Hospital,

College of Medicine, The

Catholic University of Korea, 271

Cheonbo-ro, Uijeongbu 11765 ,

Korea

Tel: $+82-31-320-3048$

Fax: +82-31-847-2127

E-mail: drbreast@catholic.ac.kr https://orcid.org/0000-0002$9982-7838$

*'These authors contributed equally to the work.
Background/Aims: We evaluated the efficacy of docetaxel and epirubicin as neoadjuvant chemotherapy in locally advanced breast cancer and assessed the predictive factors for response to neoadjuvant chemotherapy and prognostic factors related to relapse-free survival.

Methods: Forty patients who received docetaxel and epirubicinas neoadjuvant chemotherapy for locally advanced breast cancer were evaluated retrospectively. Neoadjuvant chemotherapy consisted of intravenous injection of $75 \mathrm{mg} / \mathrm{m}^{2}$ docetaxel and $60 \mathrm{mg} / \mathrm{m}^{2}$ epirubucin on day 1, every 21 days, and two to six cycles.

Results: Twenty-five (62.5\%) patients showed a partial response, and 15 (37.5\%) patients showed a stable disease in the first response evaluation after two or three cycles of neoadjuvant chemotherapy. In the second response evaluation of nine patients who received six cycles of neoadjuvant chemotherapy, one patient achieved a complete response, but two patients with hormone receptor-negative, human epidermal growth factor receptor 2-positive breast cancer experienced disease progression. Twenty-five (62.5\%) patients experienced downstaging after neoadjuvant chemotherapy. Patients with $>20 \%$ pretreatment Ki- 67 and decrease of Ki- 67 between pre- and post-neoadjuvant chemotherapy showed a trend for better response. In multivariate analysis, advanced pathological stage showed a significant negative effect on relapse-free survival.

Conclusions: Docetaxel and epirubicin neoadjuvant chemotherapy showed a good response in locally advanced breast cancer. Pretreatment Ki-67 and change of Ki67 may play a role as predictive factor for response to neoadjuvant chemotherapy.

Keywords: Breast neoplasms; Chemotherapy; Docetaxel; Epirubicin; Ki-67

\section{INTRODUCTION}

Breast cancer is the second most common cancer in Korean women. According to the Korea Central Cancer Registry, in 2014, 33.9\% and $9.2 \%$ of breast cancer patients were diagnosed at stage II and III, respectively; i.e., about one-third of patients have locally advanced breast cancer at diagnosis. The use of neoadjuvant chemotherapy (NAC) in locally advanced breast cancer has some clinical advantages. First, NAC can reduce the tumor size and make patients more suitable for surgical resection. Second, randomized clinical trials have shown no significant differences in long-term outcomes between neoadjuvant and adjuvant chemotherapy [1]. In other 
words, it can eradicate micrometastases and reduce the incidence of recurrence to the same extent as adjuvant chemotherapy. Third, use of NAC may provide important prognostic information and allow monitoring of the treatment response. Achieving a pathological complete response (pCR) to NAC is associated with favorable relapse-free survival (RFS) and overall survival (OS) in early-stage breast cancer [1].

Anthracyclines and taxanes are the representative chemotherapeutic agents used in breast cancer. It is known that the addition of a taxane to ananthracycline-based regimen can increase its response rate and efficacy. The combination of docetaxel and epirubicin (DE) is one of the NAC that is used for locally advanced breast cancer. Despite the relatively high response rate to an anthracycline in combination with a taxane, it is clear that some patients have no response or experience disease progression. In this study, we aimed to analyze the clinical outcome of using docetaxel and epirubicin neoadjuvant chemotherapy (DE NAC) and to investigate the difference in the clinical characteristics of responders and nonresponders.

\section{METHODS}

\section{Patients}

Forty patients who received DE NAC for locally advanced breast cancer at our institution between January 2009 and December 2016 were included. All patients had disease pathologically confirmed by core needle biopsy of the main mass or axillary lymph nodes before NAC. Clinical records and pathology reports were reviewed retrospectively. The following clinical data were collected: age, sex, comorbidities, tumor stage, surgery, pathology results including estrogen receptor, progesterone receptor, and human epidermal growth factor receptor 2 (HER2), chemotherapy, radiotherapy, recurrence and survival. Ethics Committee approval was obtained from the Institutional Review Board of The Catholic University of Korea, Uijeongbu St. Mary's Hospital (IRB No. UC18RESIoo02). Written informed consent was waived due to a retrospective nature of our study.

\section{Neoadjuvant chemotherapy and response evaluation}

Patients underwent mammography and breast sonog- raphy as an initial work-up. After pathological confirmation of breast cancer, breast magnetic resonance imaging, bone scan, and ${ }^{18} \mathrm{~F}$-fluorodeoxyglucose positron-emission tomography-computed tomography were performed for staging. The NAC consisted of intravenous injection of $75 \mathrm{mg} / \mathrm{m}^{2}$ docetaxel and $60 \mathrm{mg} / \mathrm{m}^{2}$ epirubicin on day 1 , then every 21 days. Patients received two to six cycles of NAC, and curative resection was performed 3 to 4 weeks after the last dose of chemotherapy. Follow-up imaging for response evaluation was performed after two or three cycles and six cycles, respectively. Response evaluation was assessed according to the Response Evaluation Criteria in Solid Tumors (version 1.1). A complete response (CR) was defined as disappearance of all target lesions, with any pathological lymph nodes having a reduction in short axis to $<10 \mathrm{~mm}$. A partial response (PR) was defined as at least a $30 \%$ decrease in the sum of the diameters of target lesions, taking as reference the baseline sum diameter. Progressive disease (PD) was defined as at least a $20 \%$ increase in the sum of the diameters of target lesions, taking as reference the smallest sum recorded. Stable disease (SD) was defined as neither PR nor PD. The pathological response was analyzed by a board-certified pathologist who was blinded to the clinical data. A pCR was defined as the absence of residual invasive disease in the breast and in the axillary lymph nodes.

\section{Statistical analysis}

Student's $t$ test was used to evaluate the significance of differences between the continuous variables. Categorical variables were compared using the chi-square and Fisher's exact tests. RFS was defined as the time from the date of surgery to the date of recurrence or censored at the last follow-up date. Univariate analysis and survival curves were estimated using the Kaplan-Meier method, and the log-rank test was applied to identify differences. Multivariate analysis was performed using Cox proportional hazards regression models, and adjusted hazard ratios with 95\% confidence intervals (CIs) were calculated. A p value of less than 0.05 was considered significant.

\section{RESULTS}

\section{Patient characteristics}

The clinicopathological characteristics of the 40 patients 
Table 1. Clinicopathological characteristics of the patients

\begin{tabular}{|c|c|}
\hline Variable & All patients $(n=40)$ \\
\hline Age, yr & $48.5(24-70)$ \\
\hline \multicolumn{2}{|l|}{ Menopausal status } \\
\hline Premenopause & $22(55.0)$ \\
\hline Postmenopause & $18(45.0)$ \\
\hline \multicolumn{2}{|l|}{ Comorbidity } \\
\hline$\geq 1$ & $8(20.0)$ \\
\hline \multicolumn{2}{|l|}{ Tumor location } \\
\hline Right & $20(50.0)$ \\
\hline Left & $19(47 \cdot 5)$ \\
\hline Both & $1(2.5)$ \\
\hline \multicolumn{2}{|l|}{ Histologic type } \\
\hline Ductal & $36(90.0)$ \\
\hline Lobular & $2(5.0)$ \\
\hline Other & $2(5.0)$ \\
\hline \multicolumn{2}{|c|}{ Estrogen receptor status } \\
\hline Positive & $20(50.0)$ \\
\hline Negative & $18(45.0)$ \\
\hline NA & $2(5.0)$ \\
\hline \multicolumn{2}{|c|}{ Progesterone receptor status } \\
\hline Positive & $16(40.0)$ \\
\hline Negative & $22(55 \cdot 0)$ \\
\hline NA & $2(5.0)$ \\
\hline \multicolumn{2}{|l|}{ HER2 status } \\
\hline Positive & $11(27.5)$ \\
\hline Negative & $19(47.5)$ \\
\hline Equivocal & $8(20.0)$ \\
\hline NA & $2(5.0)$ \\
\hline TNBC & $10(25.0)$ \\
\hline \multicolumn{2}{|l|}{$\mathrm{Ki}-67$} \\
\hline$>20 \%$ & $27(67 \cdot 5)$ \\
\hline \multicolumn{2}{|l|}{ P53 status } \\
\hline Positive & $16(40.0)$ \\
\hline \multicolumn{2}{|l|}{ Clinical stage } \\
\hline IIB & $2(5.0)$ \\
\hline IIIA & $15(37 \cdot 5)$ \\
\hline IIIB & $11(27.5)$ \\
\hline IIIC & $12(30.0)$ \\
\hline \multicolumn{2}{|l|}{ Clinical T stage } \\
\hline $\mathrm{T} 1$ & $1(2.5)$ \\
\hline $\mathrm{T} 2$ & $10(25.0)$ \\
\hline $\mathrm{T}_{3}$ & $10(25.0)$ \\
\hline $\mathrm{T}_{4}$ & $19(47 \cdot 5)$ \\
\hline \multicolumn{2}{|l|}{ Clinical N stage } \\
\hline $\mathrm{N} 1$ & $6(15.0)$ \\
\hline $\mathrm{N} 2$ & $22(55.0)$ \\
\hline $\mathrm{N}_{3}$ & $12(30.0)$ \\
\hline
\end{tabular}

Values are presented as median (range) or number (\%). NA, not available; HER2, human epidermal growth factor receptor 2; TNBC, triple-negative breast cancer.
Table 2. Exposure to neoadjuvant docetaxel/epirubicin chemotherapy

\begin{tabular}{lc}
\hline Variable & $\begin{array}{c}\text { All patients } \\
(\mathrm{n}=40)\end{array}$ \\
\hline $\begin{array}{l}\text { No. of cycle, median }(\mathrm{range}) \\
\text { Cumulative dose, } \mathrm{mg} / \mathrm{m}^{2}, \text { mean } \pm \mathrm{SD}\end{array}$ & $3(2-6)$ \\
\multicolumn{1}{c}{ Docetaxel } & $251.7 \pm 79.8$ \\
$\quad$ Epirubicin & $201.3 \pm 63.9$ \\
Relative dose intensity, \% & \\
Mean \pm SD & $91.5 \pm 5.2$ \\
\hline Median (range) & $90.5(81-100)$ \\
\hline
\end{tabular}

$\mathrm{SD}$, standard deviation.

Table 3. Response rates of neoadjuvant chemotherapy

\begin{tabular}{lc}
\hline Response & All patients $(\mathrm{n}=40)$ \\
\hline Clinical response & $1(2.5)$ \\
CR & $24(60.0)$ \\
PR & $13(32.5)$ \\
SD & $2(5.0)$ \\
PD & \\
Pathological response & $2(5.0)$ \\
\hline
\end{tabular}

Values are presented as number (\%).

$\mathrm{CR}$, complete response; $\mathrm{PR}$, partial response; $\mathrm{SD}$, stable disease; $\mathrm{PD}$, progressive disease.

are summarized in Table 1. The mean age of the patients was 48.5 years (range, 24 to 70 ), and 22 patients (55.0\%) were premenopausal. The median primary tumor diameter before treatment was $5.7 \mathrm{~cm} ; 28$ (70.0\%) patients had tumors larger than $5 \mathrm{~cm}$ in diameter. All patients except two were clinical stage III. Twenty-eight (70\%) patients received three cycles of NAC, and nine $(22.5 \%)$ received six cycles. The mean relative dose intensity for DE was 91.5\% (Table 2). After NAC, modified radical mastectomy was performed in $35(87.5 \%)$ patients and breast conserving surgery in five (12.5\%).

\section{Clinical outcome of neoadjuvant chemotherapy}

Based on the first response evaluation after two or three cycles of NAC, 25 (62.5\%) patients showed a PR, and 15 (37.5\%) patients had SD. In the second response evaluation of the nine patients who received six cycles of NAC, one patient achieved a CR, but two had PD. The results 
Table 4. Clinicopathological characteristics according to response of neoadjuvant chemotherapy

\begin{tabular}{|c|c|c|c|}
\hline Variable & $\mathrm{CR} / \mathrm{PR}(\mathrm{n}=25)$ & $\mathrm{SD} / \mathrm{PD}(\mathrm{n}=15)$ & $p$ value \\
\hline Menopausal status & & & 0.870 \\
\hline Pre & $14(56)$ & $8(53 \cdot 3)$ & \\
\hline Post & $11(44)$ & $7(46.7)$ & \\
\hline HR status & & & 0.935 \\
\hline Positive & $13(52)$ & $8(53 \cdot 3)$ & \\
\hline Negative & $12(48)$ & $7(46.7)$ & \\
\hline HER2 status $(\mathrm{n}=30)$ & & & 0.719 \\
\hline Positive & $8(42.1)$ & $3(27 \cdot 3)$ & \\
\hline Negative & $11(57 \cdot 9)$ & $8(72.7)$ & \\
\hline TNBC & $6(24)$ & $4(26.7)$ & 0.850 \\
\hline Non-TNBC & $19(76)$ & $11(73 \cdot 3)$ & \\
\hline $\mathrm{Ki}-67(\mathrm{n}=37)$ & & & 0.091 \\
\hline$>20 \%$ & $19(82.6)$ & $8(57.1)$ & \\
\hline$\leq 20 \%$ & $4(17 \cdot 4)$ & $6(42.9)$ & \\
\hline $\mathrm{P}_{53}$ status $(\mathrm{n}=37)$ & & & 0.987 \\
\hline Positive & $10(43.5)$ & $6(42.9)$ & \\
\hline Negative & $13(56.5)$ & $8(57.1)$ & \\
\hline Tumor size, $\mathrm{cm}$ & & & 0.680 \\
\hline$\leq 5.0$ & $10(40)$ & $7(46.7)$ & \\
\hline$>5.0$ & $15(60)$ & $8(53.5)$ & \\
\hline Clinical stage & & & 0.430 \\
\hline IIB & $2(8)$ & 0 & \\
\hline IIIA & $10(40)$ & $5(33 \cdot 3)$ & \\
\hline IIIB & $5(20)$ & $6(40)$ & \\
\hline IIIC & $8(32)$ & $4(26.7)$ & \\
\hline Ki-67 change $(\mathrm{n}=37)^{\mathrm{a}}$ & & & 0.015 \\
\hline Yes & $16(69.6)$ & $4(28.6)$ & \\
\hline No & $7(30.4)$ & $10(71.4)$ & \\
\hline
\end{tabular}

CR, complete response; PR, partial response; SD, stable disease; PD, progressive disease; HR, hormone receptor; HER2, human epidermal growth factor receptor 2; TNBC, triple-negative breast cancer.

${ }^{a}$ Ki- 67 change means as pre-chemotherapy Ki- $67>20 \%$ and post-chemotherapy Ki- $67 \leq 20 \%$.

of the final response assessment performed before surgery are summarized in Table 3. The two patients who had PD at the second evaluation had hormone receptor (HR)-negative/HER2-positive breast cancer.

Two (5.0\%) of the 40 patients had a pCR, one with triple-negative breast cancer and one with HR-positive/ HER2-positive breast cancer. In the neoadjuvant pathologic staging (ypTNM), the numbers of patients with stage o, IA, IIA, IIB, IIIA, and IIIC were 2 (5\%), 6 (12\%), 9 (22.5\%), 2 (5\%), 10 (25\%), and $11(27.5 \%)$, respectively. Twen- ty-five (62.5\%) patients were down-staged after NAC.

There was no difference in the response rate according to breast cancer subtype. Patients with $>20 \% \mathrm{Ki}-67$ labeling index pretreatment tended to have a better response to NAC. A decrease of Ki-67 labeling index between pre- and post-NAC was significantly more frequent in patients who had CR or PR (Table 4).

\section{Recurrence and survival analysis}

The median follow-up was 37.3 months (range, 6.4 to 
Table 5. Univariate survival analyses according to clinicopathological variables

\begin{tabular}{|c|c|c|}
\hline \multirow{2}{*}{ Variable } & \multicolumn{2}{|c|}{ All patients $(n=40)$} \\
\hline & 3-year RFS, \% & $p$ value \\
\hline Clinical stage & & 0.025 \\
\hline IIB & 100 & \\
\hline IIIA/B & 84.6 & \\
\hline IIIC & 50.0 & \\
\hline Tumor size, cm & & 0.061 \\
\hline$\leq 5.0$ & 82.3 & \\
\hline$>5.0$ & 69.5 & \\
\hline Clinical N stage & & 0.011 \\
\hline $\mathrm{N} 1 / 2$ & 85.7 & \\
\hline $\mathrm{N}_{3}$ & 50.0 & \\
\hline Pathological stage & & 0.015 \\
\hline O-IIB & 100 & \\
\hline IIIA/B & 60.0 & \\
\hline IIIC & $45 \cdot 4$ & \\
\hline Pathological T stage & & 0.001 \\
\hline $\mathrm{T} 1$ & 82.3 & \\
\hline $\mathrm{T} 2$ & 82.3 & \\
\hline $\mathrm{T}_{3}$ & $33 \cdot 3$ & \\
\hline Pathological N stage & & 0.003 \\
\hline $\mathrm{Nl} / 2$ & 89.7 & \\
\hline $\mathrm{N}_{3}$ & 36.4 & \\
\hline Grade & & 0.057 \\
\hline $\mathrm{Gl} / 2$ & 100 & \\
\hline $\mathrm{G}_{3}$ & 41.2 & \\
\hline LVI & & 0.007 \\
\hline No & 100 & \\
\hline Yes & 60.0 & \\
\hline Skin invasion & & 0.047 \\
\hline No & 82.1 & \\
\hline Yes & 50.0 & \\
\hline Down staging & & 0.041 \\
\hline No & 84.0 & \\
\hline Yes & 60.0 & \\
\hline Ki-67 change & & 0.064 \\
\hline Decreased & 82.1 & \\
\hline No change/Increased & 55.5 & \\
\hline
\end{tabular}

RFS, relapse-free survival; LVI, lymphovascular invasion.
108.6). Thirty-seven patients received adjuvant chemotherapy. Patients with HER2-positive breast cancer whose tumor with $\geq 1 \mathrm{~cm}$ in diameter or who had axillary lymph node metastasis received adjuvant trastuzumab for 1 year. All patients with HR-positive breast cancer received adjuvant hormonal therapy, and 33 also received adjuvant radiotherapy. Among 40 patients, recurrence occurred in 12, with one experiencing locoregional recurrence, seven having distant metastases, and four having both. Ten of 12 patients with recurrent disease underwent biopsy at the site of recurrence. Seven patients had the same estrogen receptor/progesterone receptor/HER2 status as at the time of initial diagnosis, but three showed a different subtype. All patients with recurrence received palliative chemotherapy or hormonal therapy.

In univariate analysis, the prognostic factors significantly affecting RFS were clinical stage, clinical N stage, pathological stage, grade, lymphovascular invasion, skin invasion, down-staging, and change between pre- and postchemotherapy Ki-67 labeling index (Table 5). In multivariate analysis, advanced pathological stage was significantly negatively associated with RFS (hazard ratio, 8.354; $95 \%$ CI, 1.305 to $53.456 ; p=0.025)$.

\section{DISCUSSION}

The use of neoadjuvant treatment for selected high-risk, locally advanced breast cancer is well established. Similar to adjuvant chemotherapy, NAC regimens most commonly contain an anthracycline (adriamycin or epirubicin) in combination or sequentially administered with a taxane (paclitaxel or docetaxel). The adriamycin and cyclophosphamide (AC) regimen is known to be highly active in the treatment of breast cancer, and is predominantly used in an adjuvant setting. In the Early Breast Cancer Trialists' Collaborative Group meta-analyses, AC adjuvant chemotherapy led to an $8.0 \%$ reduction in the 10-year recurrence rate compared with no chemotherapy [2]. The results from the National Surgical Adjuvant Breast and Bowel Project (NSABP) B-28 and Cancer And Leukemia Group B 9344 demonstrated that the addition of a taxane to adjuvant AC significantly improved the RFS and OS in patients with axillary lymph node-positive breast cancer $[3,4]$. In the NSABP B-18 trial, there was 
no significant difference in OS between the adjuvant AC and neoadjuvant AC groups [5]. The NSABP B-27 trial was conducted in three groups: AC followed by surgery, AC followed by taxane (AC-T) and then surgery, and AC followed by surgery and then taxane. The neoadjuvant AC-T regimen showed a higher $\mathrm{PCR}$ rate compared with the neoadjuvant $\mathrm{AC}$ regimen, and $\mathrm{PCR}$ was a significant predictor of OS regardless of treatment [6]. The results of these clinical trials suggest that NAC has equivalent efficacy to adjuvant chemotherapy, and AC-T is widely used in both adjuvant and neoadjuvant settings. The National Comprehensive Cancer Network guideline also recommended AC-T as the preferred NAC regimen in HER2-negative breast cancer.

The simultaneous administration of an anthracycline and a taxane, e.g., DE, may have some advantages compared with their sequential administration. First, simultaneous administration of two potent agents can be expected to induce a high response rate. Second, the AC-T regimen is administered every 21 days for eight cycles, while the DE regimen is administered every 21 days for three to six cycles. Thus, the period from diagnosis to surgery is shorter in patients treated with DE. In addition, it can be easy to identify an early clinical response postoperative chemotherapy and thereby clarify the prognosis. There are previous reports of the efficacy and safety of DE in breast cancer. Sessa et al. [7] reported that DE showed an overall response rate of $66 \%$ in patients with advanced breast cancer. Hirano et al. [8] reported that DE had a clinical response rate of $90.5 \%$ as NAC in locally-advanced breast cancer: two of the 21 patients in that study achieved pCR. In our study, $62.5 \%$ of patients showed a clinical CR or PR, and two achieved a pCR. Our results do not differ from those of previous studies: in the setting of combined treatment with anthracycline and docetaxel, clinical response and pCR rates range from $68 \%$ to $93 \%$ and from $8 \%$ to $16 \%$, respectively [9].

In our study, two patients experienced disease progression after six cycles of chemotherapy, despite showing a response after three cycles. Both patients had HR-negative/HER2-positive breast cancer. This suggests that an early start of anti-HER2 targeted therapy is important for the clinical outcome in HER2-positive breast cancer. Currently, regimens including a combination of HER2-targeting agents such as trastuzumab and/ or pertuzumab are widely used as NAC in HER2-pos- itive breast cancer. However, in the early 2010 s in Korea, it was not easy to use anti-HER2 targeted therapy in a neoadjuvant setting because of issues with cost reimbursement by the health system. Thus, patients with HER2-positive breast cancer, including those in our study, did not receive anti-HER2 targeted therapy as part of the NAC. In the NeOAdjuvant Herceptin (NOAH) trial, NAC with trastuzumab followed by adjuvant trastuzumab increased the rate of PCR and significantly improved event-free survival compared with NAC alone in patients with HER2-positive locally advanced breast cancer [10]. Palmieri et al. [11] compared the efficacy of NAC plus trastuzumab with that of NAC alone followed by adjuvant trastuzumab. The administration of trastuzumab in both the neoadjuvant and adjuvant periods had the greatest positive effect in patients with HR-negative/HER2-postive breast cancer [11]. These data and our results indicate the necessity of the early introduction of anti-HER2 targeted therapy, especially in HR-negative/HER2-positive breast cancer.

There have been previous studies on prognostic and predictive factors related to NAC in locally advanced breast cancer. The most well-known factors are breast cancer subtypes, pCR and Ki-67 labeling index. In neoadjuvant clinical trials with HER2-targeted agents, the proportion of patients achieving a pCR was higher in patients with HR-negative disease than in those with HR-positive disease [12,13]. Faneyte et al. [14] analyzed the clinicopathological factors to predict response of the primary tumor in 97 patients who received anthracycline-based NAC. They showed that proliferation decreases significantly after chemotherapy, ER negativity and a high proliferation index were associated with better response. Gillon et al. [15] reported the breast cancer subtype classification and pathological response as predictors of locoregional recurrence in patients treated with NAC. Triple negative breast cancer and lack of pathological response were associated with higher locoregional recurrence risk. Li et al. [16] reported the results of meta-analysis that patients who achieved a pCR after NAC had significantly lower rates of local and distant RFS.

Ki-67 is a nuclear antigen expressed during the $\mathrm{G}_{1}, \mathrm{~S}$, $\mathrm{G} 2$, and $\mathrm{M}$ phases of the cell cycle, but not during the Go phase. It has been established as a marker of proliferation in various cancers [17]. During the last decade, 
several studies have focused on the prognostic and predictive value of Ki-67 expression by tumor cells in the setting of neoadjuvant treatment of breast cancer. Nishimura et al. [18] reported that higher Ki-67 labeling before NAC was significantly related to higher $\mathrm{pCR}$ rates and that lower Ki-67 labeling after NAC was associated with a better prognosis. Dowsett et al. [19] reported that higher Ki-67 labeling index after 2 weeks of neoadjuvant hormone treatment was associated with shorter RFS. This suggests that the change in Ki-67 labeling 2 weeks after the initial endocrine treatment is a good predictor of RFS. Tao et al. [20] reported the results of meta-analysis for Ki-67 labeling index as a predictive marker for a pCR to NAC. Pretherapeutic Ki-67 labeling index was associated with pCR in breast cancer patients undergoing NAC. In our study, patients with $>20 \% \mathrm{Ki}-$ 67 labeling index before NAC tended to have better responses. This indicates that the Ki- 67 value before NAC is a strong predictive factor for the effectiveness of the therapy. In addition, patients with Ki-67 labeling > 20\% before NAC that decreased to $\leq 20 \%$ after NAC tended to have better RFS. In univariate survival analyses, clinical and pathological staging, lymphovascular invasion, skin invasion, and down-staging after NAC were significantly associated with RFS. Only pathological staging showed a significant association with RFS in multivariate analysis. Our study has certain limitations with small number of patients and short follow-up periods. We could not find the differences in tumor response and prognosis according to breast cancer subtype due to limited sample sizes. It is also impossible to properly evaluate the impact of achievement of pCR on prognosis. Although there are limitations, it can be worth as the additional data of neoadjuvant docetaxel and epirubicin from clinical practice in locally advanced breast cancer.

In conclusion, DE combination chemotherapy induced a good response, with a rate of $62.5 \% \mathrm{CR}$ and $\mathrm{PR}$, and can therefore be one option for NAC in locally advanced HER2-negative breast cancer. Neoadjuvant treatment including anti-HER2 targeted therapy is very important for improving the prognosis in HER2-positive breast cancer. Pretreatment Ki- 67 labeling index and the change in $\mathrm{Ki}-67$ labeling after NAC are likely to act as predictive and prognostic factors in breast cancer. Further research is needed to clarify how Ki-67 labeling index could influence clinical decisions in the neoadju- vant setting. These studies should be continued to identify the group of patients who will benefit from NAC.

\section{KEY MESSAGE}

1. Docetaxel and epirubicin combination chemotherapy can be considered as one option for neoadjuvant chemotherapy (NAC) in locally advanced human epidermal growth factor receptor 2 (HER2)-negative breast cancer.

2. Two patients with hormone receptor (HR)-negative, HER2-positive breast cancers experienced disease progression after 6 cycles of NAC. It suggests the necessity of the early introduction of anti-HER2 targeted therapy in HR-negative/HER2-positive breast cancer.

3. Pretreatment Ki-67 labeling index and the change in Ki-67 labeling after NAC are likely to act as predictive and prognostic factors in breast cancer.

\section{Conflict of interest}

No potential conflict of interest relevant to this article was reported.

\section{Acknowledgments}

This work was supported by the Basic Science Research Program through the National Research Foundation of Korea (NRF) funded by the Ministry of Education (NRF-2016R1DiA1B03933245)(Hye Sung Won).

\section{REFERENCES}

1. Thompson AM, Moulder-Thompson SL. Neoadjuvant treatment of breast cancer. Ann Oncol 2012;23:X231-X236.

2. Early Breast Cancer Trialists' Collaborative Group (EBCTCG). Effects of chemotherapy and hormonal therapy for early breast cancer on recurrence and 15-year survival: an overview of the randomised trials. Lancet 2005;365:1687-1717.

3. Mamounas EP, Bryant J, Lembersky B, et al. Paclitaxel after doxorubicin plus cyclophosphamide as adjuvant chemotherapy for node-positive breast cancer: results from NSABP B-28. J Clin Oncol 2005;23:3686-3696. 
4. Henderson IC, Berry DA, Demetri GD, et al. Improved outcomes from adding sequential paclitaxel but not from escalating doxorubicin dose in an adjuvant chemotherapy regimen for patients with node-positive primary breast cancer. J Clin Oncol 2003;21:976-983.

5. Wolmark N, Wang J, Mamounas E, Bryant J, Fisher B. Preoperative chemotherapy in patients with operable breast cancer: nine-year results from National Surgical Adjuvant Breast and Bowel Project B-18. J Natl Cancer Inst Monogr 2001:96-102.

6. Bear HD, Anderson S, Smith RE, et al. Sequential preoperative or postoperative docetaxel added to preoperative doxorubicin plus cyclophosphamide for operable breast cancer: National Surgical Adjuvant Breast and Bowel Project Protocol B-27. J Clin Oncol 2006;24:2019-2027.

7. Sessa C, Pagani O. Docetaxel and epirubicin in advanced breast cancer. Oncologist 2001;6:13-16.

8. Hirano A, Shimizu T, Imamura H, et al. The combination of epirubicin plus docetaxel as neoadjuvant chemotherapy in locally-advanced breast cancer. Anticancer Res 2006;26:581-584.

9. Papadimitriou K, Ardavanis A, Kountourakis P. Neoadjuvant therapy for locally advanced breast cancer: focus on chemotherapy and biological targeted treatments' armamentarium. J Thorac Dis 2010;2:160-170.

10. Gianni L, Eiermann W, Semiglazov V, et al. Neoadjuvant chemotherapy with trastuzumab followed by adjuvant trastuzumab versus neoadjuvant chemotherapy alone, in patients with HER2-positive locally advanced breast cancer (the NOAH trial): a randomised controlled superiority trial with a parallel HER2-negative cohort. Lancet 2010;375:377-384.

11. Palmieri C, Macpherson IR, Yan K, et al. Neoadjuvant chemotherapy and trastuzumab versus neoadjuvant chemotherapy followed by post-operative trastuzumab for patients with HER2-positive breast cancer. Oncotarget 2016;7:13209-13220.

12. Gianni L, Pienkowski T, Im YH, et al. Efficacy and safety of neoadjuvant pertuzumab and trastuzumab in women with locally advanced, inflammatory, or early HER2-positive breast cancer (NeoSphere): a randomised multicentre, open-label, phase 2 trial. Lancet Oncol 2012;13:25-32.

13. Hurvitz SA, Martin M, Symmans WF, et al. Neoadjuvant trastuzumab, pertuzumab, and chemotherapy versus trastuzumab emtansine plus pertuzumab in patients with HER2-positive breast cancer (KRISTINE): a randomised, open-label, multicentre, phase 3 trial. Lancet Oncol 2018;19:115-126.

14. Faneyte IF, Schrama JG, Peterse JL, Remijnse PL, Rodenhuis S, van de Vijver MJ. Breast cancer response to neoadjuvant chemotherapy: predictive markers and relation with outcome. Br J Cancer 2003;88:406-412.

15. Gillon P, Touati N, Breton-Callu C, Slaets L, Cameron D, Bonnefoi H. Factors predictive of locoregional recurrence following neoadjuvant chemotherapy in patients with large operable or locally advanced breast cancer: an analysis of the EORTC 10994/BIG 1-oo study. Eur J Cancer 2017;79:226-234.

16. Li X, Dai D, Chen B, Tang H, Wei W. Oncological outcome of complete response after neoadjuvant chemotherapy for breast conserving surgery: a systematic review and meta-analysis. World J Surg Oncol 2017;15:210.

17. von Minckwitz G, Schmitt WD, Loibl S, et al. Ki67 measured after neoadjuvant chemotherapy for primary breast cancer. Clin Cancer Res 2013;19:4521-4531.

18. Nishimura R, Osako T, Okumura Y, Hayashi M, Arima N. Clinical significance of Ki-67 in neoadjuvant chemotherapy for primary breast cancer as a predictor for chemosensitivity and for prognosis. Breast Cancer 2010;17:269275 .

19. Dowsett M, Smith IE, Ebbs SR, et al. Prognostic value of Ki67 expression after short-term presurgical endocrine therapy for primary breast cancer. J Natl Cancer Inst 2007;99:167-170.

20. Tao M, Chen S, Zhang X, Zhou Q. Ki-67 labeling index is a predictive marker for a pathological complete response to neoadjuvant chemotherapy in breast cancer: a meta-analysis. Medicine (Baltimore) 2017;96:e9384. 